\title{
Clinicoradiological changes of brain NK/T cell lymphoma manifesting pure akinesia: a case report
}

\author{
Susumu Ishihara', Osamu Kano ${ }^{2}$, Ken Ikeda ${ }^{2 *}$, Reiko Shimokawa ${ }^{3}$, Kiyokazu Kawabe ${ }^{2}$ and Yasuo Iwasaki
}

\begin{abstract}
Background: Pure akinesia (PA) is a distinct form of parkinsonism characterized by freezing phenomena. Little is known about brain tumor-associated PA. We highlight the clinicoradiological changes in a patient with PA and central nervous system (CNS) metastases of natural killer/T-cell lymphoma (NKTL).

Case presentation: A 68-year-old man with stage IVB extranodal NKTL developed a gait disturbance. Neurological examination of his gait revealed freezing, start hesitation, short step, forward flexion posture, festination and postural instability. Mild facial hypomimia and micrographia were observed. There was no rigidity or tremor in any of the four extremities. Brain magnetic resonance imaging (MRI) displayed T2-hyperintense lesions in the dorsal brainstem, cerebellum and periventricular white matter. Diffusion-weighted imaging (DWI) and the apparent diffusion coefficient (ADC) revealed hyperintensity in these regions. Cerebrospinal fluid cytology revealed CD56positive cells on immunohistochemical staining. The patient's neurological deficits did not respond to L-dopa treatment and intrathecal administration of methotrexate (MTX). Two weeks later, he displayed confusion and generalized convulsions. T2-hyperintense lesions spread to the basal ganglia and the infratentorial regions. Gadolinium enhancement was observed in the cerebellum and frontal subcortex. DWI and the ADC revealed diffusion-restricted lesions in the middle cerebellar peduncles, left internal capsules and cerebral white matter. MTX pulse therapy and intrathecal administration of cytosine arabinoside and MTX were performed. Two months later, his ambulatory state was normalized. Brain MRI also revealed marked alleviation of the infratentorial and supratentorial lesions.

Conclusions: The clinicoradiological profile of our patient suggested that dorsal ponto-mesencephalic lesions could contribute to the pathogenesis of PA. Physicians should pay more attention to striking CNS seeding of metastatic NKTL. MTX pulse therapy had an excellent effect in improving serious symptoms and brain lesions in our patient.
\end{abstract}

\section{Background}

Pure akinesia (PA) is a distinct form of parkinsonism that was first reported in 1972 as freezing phenomena only, characterized by frozen gait, micrographia and festinating speech [1]. Limb rigidity and tremor were not observed. L-dopa treatment had no effects in PA patients [2,3]. Many studies of neoplastic parkinsonism have been reported [4-14]. However, little is known about brain tumor-associated PA [4,5]. We highlight the

\footnotetext{
* Correspondence: keni@med.toho-u.ac.jp

${ }^{2}$ Department of Neurology, Toho University Omori Medical Center, Tokyo, Japan

Full list of author information is available at the end of the article
}

clinicoradiological changes in a unique patient with PA and central nervous system (CNS) metastasis of natural killer/T cell lymphoma (NKTL).

\section{Case presentation}

A 68-year-old man developed anorexia and exhibited body weight loss for 2 months. Abdominal computed tomography showed massive lesions in the adrenal glands. Laparoscopic biopsy of the adrenal gland demonstrated a pathological diagnosis of extranodal NKTL (Figure 1). Clinical stage IVB was confirmed. Two courses of DeVIC (carboplatin, etoposide, ifosfamide and dexamethasone) combination chemotherapy

\section{Biomed Central}




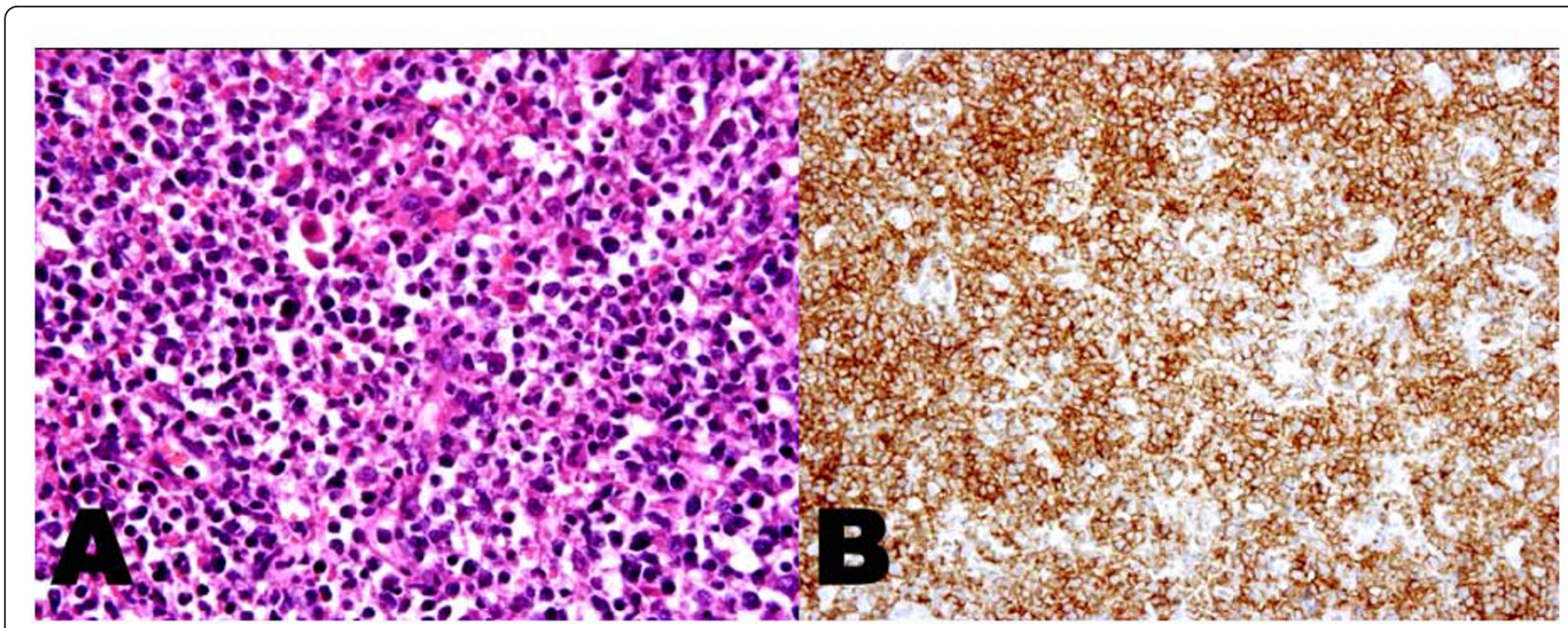

Figure 1 Biopsy specimen of the adrenal gland. A. Hematoxylin and eosin staining. Lymphoid cells are characterized by an intermediate size and nuclei with irregular contours and inconspicuous nucleoli. Original magnification $\times 200$. B. Immunohistochemical CD56 staining. Most cell membranes are CD56 positive. Original magnification $\times 200$.

led to marked amelioration of the pericardial, pulmonary and pancreatic lesions. Two weeks later, the patient developed a gait disturbance and came to our neurology department. Physical examination showed blood pressure of $120 / 78 \mathrm{~mm} \mathrm{Hg}$ and body temperature of $36.2^{\circ} \mathrm{C}$. His level of consciousness and cognitive function were normal. Neurological examination revealed a frozen gait with start hesitation, short step, forward flexion posture, festination and postural instability. Kinesia paradoxale was observed when obstacles were placed in his path. He had mild facial hypomimia, reduced blinking, slight slurred speech and micrographia. There was no limb rigidity or tremor in the four extremities. The remaining examination was normal. These neurological observations supported the clinical diagnosis of PA.

Routine laboratory studies were normal. Serum soluble interleukin-2 receptor levels increased to $1850 \mathrm{U} / \mathrm{mL}$ (normal range: 220-530). There were no paraneoplastic antibodies, including antineuronal nuclear antibody types 1, 2 and 3. Serum anti-human immunodeficiency virus antibodies were not detected. Cerebrospinal fluid (CSF) analyses showed a mononuclear cell count of $28 / \mathrm{mm}^{3}$ and total protein levels of $138 \mathrm{mg} / \mathrm{dL}$. CSF cytology revealed CD56-positive cells on immunohistochemical staining. Infectious pathogen tests for herpes simplex, tuberculosis, bacteria and fungus were all negative. Brain magnetic resonance imaging (MRI) revealed T2-hyperintense lesions in the dorsal brainstem, cerebellum and periventricular white matter. Abnormal T2-intensities were not found in the basal ganglia (Figure 2). Diffusionweighted imaging (DWI) and the apparent diffusion coefficient (ADC) revealed a mild to moderate degree of hyperintensity in the brainstem and cerebellum.
Neither intrathecal administration of cytosine arabinoside (Ara-C), methotrexate (MTX) and prednisolone (PSL), nor L-dopa treatment (levodopa $300 \mathrm{mg} /$ day and carbidopa $30 \mathrm{mg} /$ day), had any effect on the patient's PA. Two weeks later, the patient experienced confusion and a generalized tonic seizure. Brain MRI revealed widespread T2-hyperintense lesions in the infratentorial region and basal ganglia (Figure 3A-D). DWI and ADC disclosed restricted water diffusivity in the middle cerebellar peduncles, brainstem, left internal capsules and cerebral white matter (Figure 3E-L). Gadolinium enhancement was found in the cerebellum and bilateral frontal subcortex. There was no abnormal enhancement in the brainstem and basal ganglia (Figure 4). The patient's level of consciousness deteriorated, and he became bedridden. After intravenous MTX pulse therapy $(4500 \mathrm{mg} /$ day) and the second intrathecal administration of Ara-C, MTX and PSL, he recovered to normal consciousness and became ambulatory with support. He had no hypertensive episodes during the entire course. Two months later, PA was ameliorated completely without L-dopa treatment. CSF cytology showed no CD56positive cells. T2-hyperintense lesions were markedly attenuated in the brainstem, basal ganglia and cerebral white matter (Figure 5). DWI and ADC showed remarkable alleviation of diffusion-restricted lesions in the middle cerebellar peduncles, brainstem and cerebral white matter.

\section{Discussion}

We reported a patient with CNS metastases of NKTL who had a transient course of PA and striking changes in the brain lesions. 


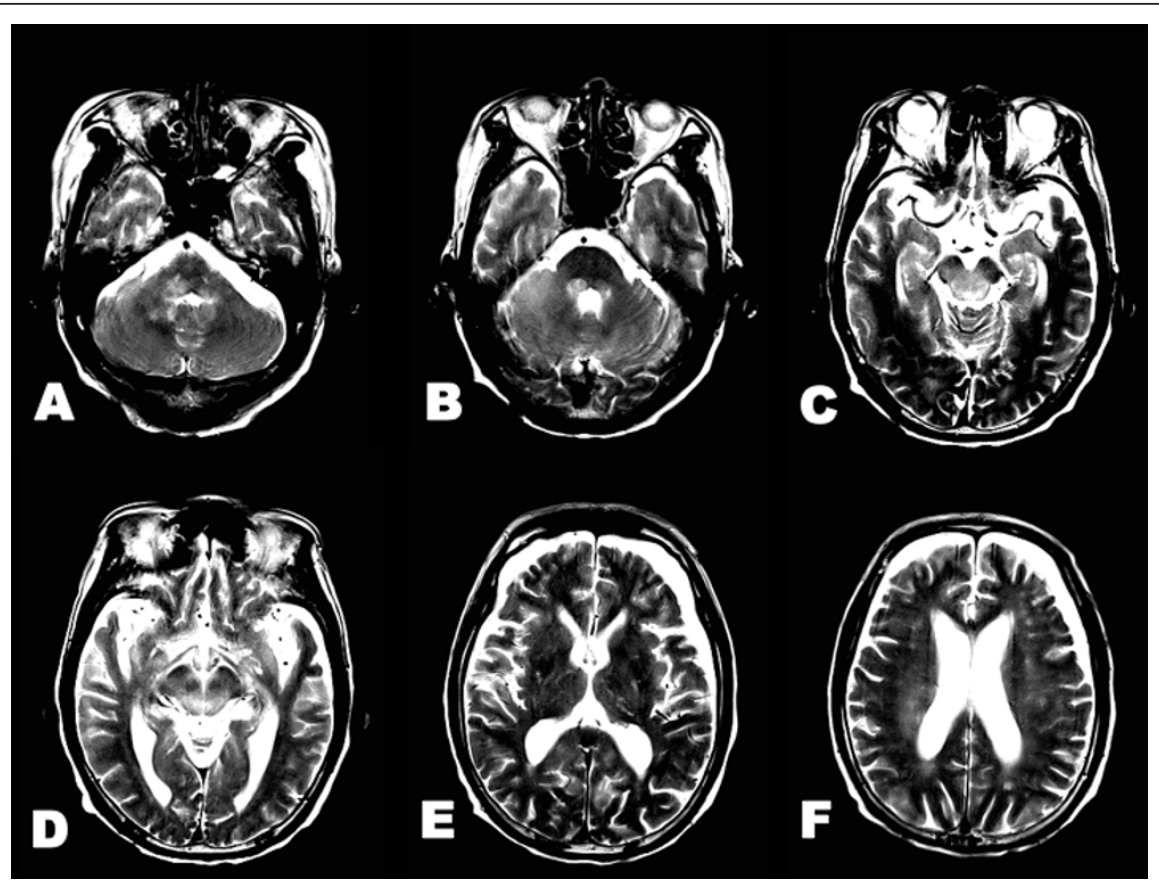

Figure 2 Brain T2-weighted imaging at neurological onset. A-F. Hyperintense lesions are observed in the dorsal pons, midbrain, cerebellum and periventricular white matter. E. There were no remarkable changes in the basal ganglia.

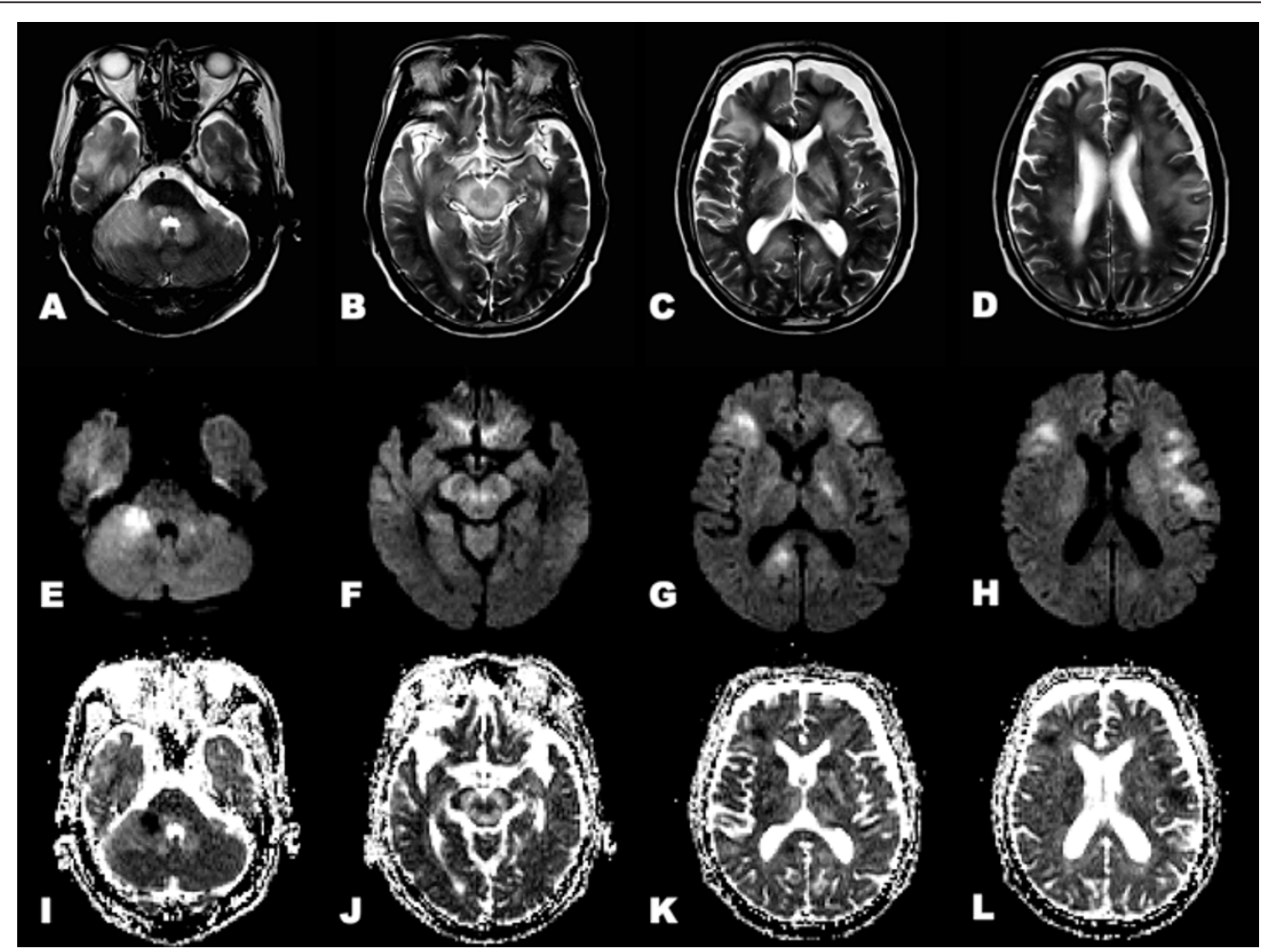

Figure 3 Brain MRI after neurological worsening. A-D. T2-weighted image show widespread T2-hyperintense lesions in the brainstem, cerebellum, basal ganglia and cerebral white matter. E-H. DWI shows hyperintense lesions in the middle cerebellar peduncles, midbrain, left internal capsules and cerebral white matter. I-L. ADC shows hypointense lesions in the middle cerebellar peduncles, midbrain, left internal capsules and cerebral white matter. 


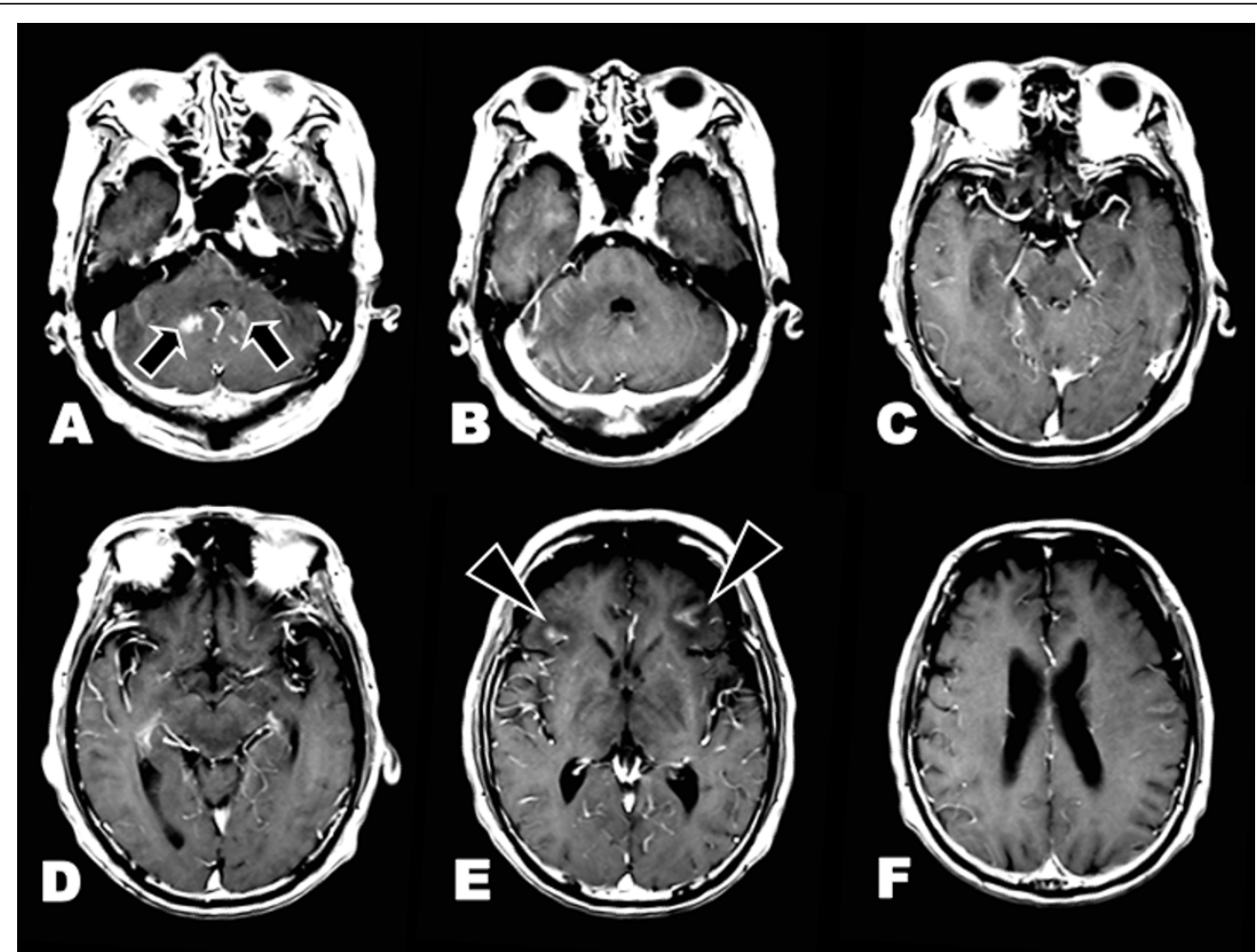

Figure 4 Gadolinium-enhanced T1-weighted imaging. A, E. Gadolinium enhancement are found in the cerebellum (arrows) and bilateral frontal subcortex (arrowheads). B-D, F. There is no enhancement in the brainstem and basal ganglia.

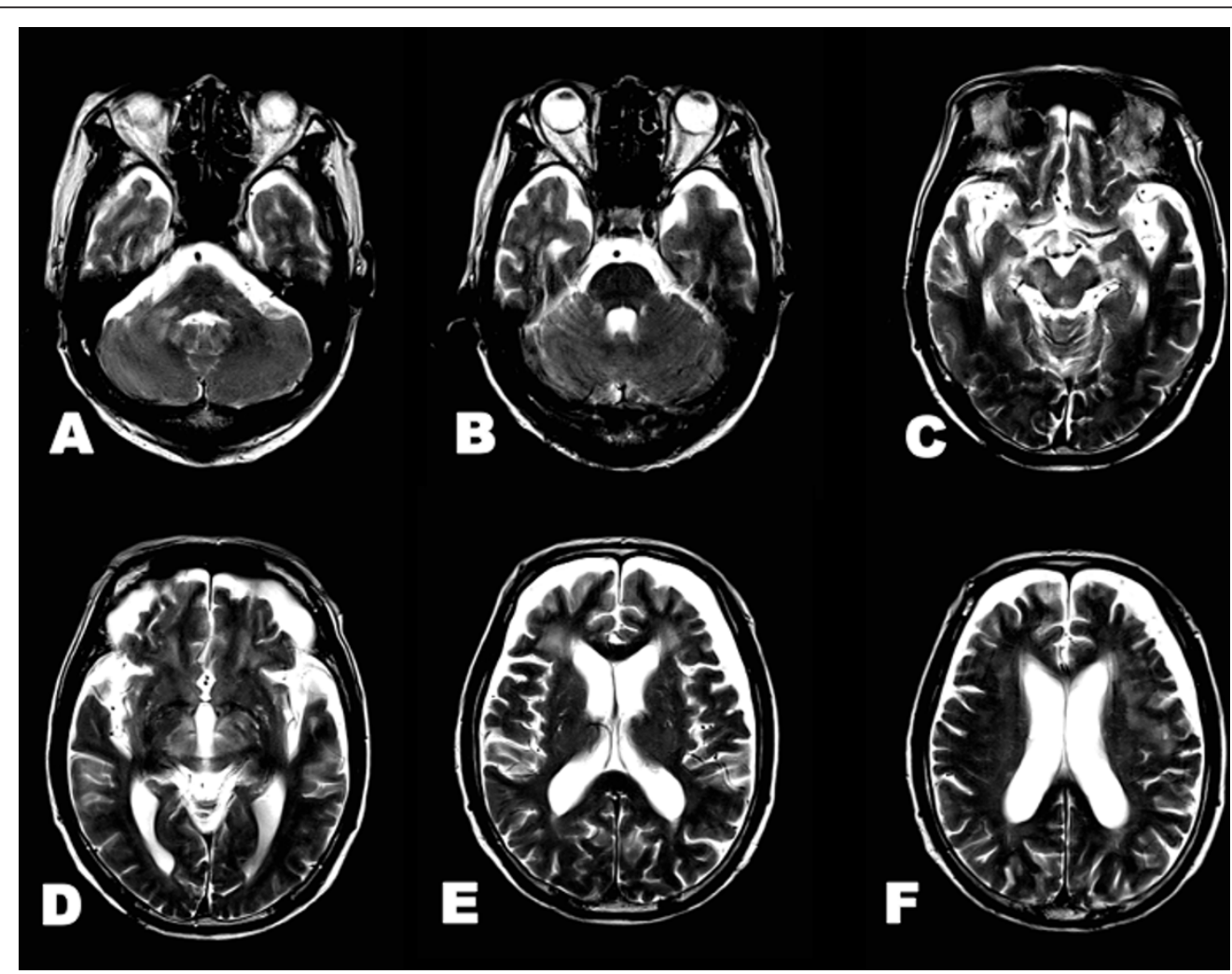

Figure 5 Brain T2-weighted imaging after neurological recovery. A-F. T2-hyperintense lesions are markedly attenuated. Hyperintense lesions remain in the right middle cerebellar peduncle, midbrain and cerebral white matter. 
The frequency of CNS metastases is $5-9 \%$ in patients with systemic non-Hodgkin lymphoma. Most patients have leptomeningeal or spinal epidural metastases. The rate of CNS parenchymal metastases is only 1\% [15]. NKTL is a rare type of lymphoma that usually presents as destructive lesions within the nasal cavity. Nasal NKTL frequently involves extranodal sites such as the lung, skin and CNS [16]. CNS invasion or metastasis is common in patients with nasal NKTL [17]. Most NKTL patients have aggressive courses and unfavorable clinical outcomes. During PA onset, our patient showed lesions in the brainstem and periventricular white matter on MRI. After he exhibited confusion and generalized convulsion, T2-hyperintense lesions were observed in the middle cerebellar peduncles and basal ganglia. DWI and the ADC revealed gadoliniumenhanced and -nonenhanced diffusion-restricted lesions in the middle cerebellar peduncles, brainstem and cerebral white matter. These neuroradiological features suggested the rapid relapse of brain NKTL. Extensive MRI lesions in the cerebral white matter have previously been described in a patient with nasal NKTL [18]. Recent neuroradiological studies have pointed out the inverse relationship between ADC and the cell density of B cell lymphoma $[19,20]$. The clinicoradiological worsening of our patient might have resulted from the fulminant progression of CNS metastases of NKTL and cerebral edema.

As mentioned previously, many studies of neoplastic parkinsonism have been reported [4-14]. The following pathogenic mechanisms underlying parkinsonism induced by brain tumors have been proposed: 1) damage of striatal postsynaptic cells in the basal ganglia or substantia nigra secondary to mechanical pressure or intrinsic involvement; 2) impairment of the pathway between the striatum and the supplementary motor area; and 3) compression and/or distortion of the nigrostriatal pathway. The topographic distribution of the brain tumors is divided into the supratentorial region and brainstem. Compared with supratentorial tumors, the frequency of brainstem tumor-related parkinsonism is rare. Previous reports of neoplastic parkinsonism associated with the midbrain lesion are summarized in Table 1 . The patterns of parkinsonism onset and the responses to Ldopa were highly variable. In these reports, the pathological diagnosis was astrocytoma in three patients and $\mathrm{B}$ cell lymphoma in three patients. The present patient was diagnosed as CNS metastases of NKTL.

With respect to the pathogenesis of PA, previous pathological studies have shown that some PA patients had similar lesions to those of progressive supranuclear palsy (PSP) patients [21-23]. Neuroradiological studies have also reported matching results between PA and PSP patients [24,25]. Only two patients with neoplastic PA have been reported [4,5]. Suzuki et al. [4] described a 44-year-old man with primary CNS reticulum cell sarcoma. Pramstaller et al. [5] reported the case of a 75year-old man with primary CNS B cell lymphoma. This patient did not respond to L-dopa treatment, and autopsy demonstrated the involvement of the bilateral globus pallidus [5]. The precise pathophysiological mechanism of PA remains unclear. In general, PA might contribute to both presynaptic and postsynaptic damage in nigrostriatal dopaminergic neurons $[24,25]$. The midbrain tegmentum is the most common lesion site between PA and PSP patients [21-23]. A dorsorostral midbrain lesion was reported to cause PSP-like symptoms in a patient who had normal nigrostriatal dopaminergic function after resection of a pineal gland tumor [26]. Therefore, the neuroradiological changes of our

Table 1 Previous literature of brainstem tumor-related parkinsonism

\begin{tabular}{|c|c|c|c|c|c|}
\hline Authors [*] (years) & Age/gender & $\begin{array}{l}\text { Histological } \\
\text { diagnosis }\end{array}$ & Tumor location & $\begin{array}{l}\text { Onset } \\
\text { form }\end{array}$ & $\begin{array}{l}\text { L-dopa } \\
\text { treatment }\end{array}$ \\
\hline Gherardit et al [9] (1985) & 59 years/male & B cell lymphoma & Midbrain, thalamus & Acute & $\begin{array}{l}\text { Excellent } \\
\text { response }\end{array}$ \\
\hline Cicarelli et al [10] (1999) & $\begin{array}{l}39 \text { years/ } \\
\text { female }\end{array}$ & Astrocytoma & Pons, midbrain & Unknown & No response \\
\hline $\begin{array}{l}\text { Yoshimura et al [11] } \\
(2002)\end{array}$ & $\begin{array}{l}63 \text { years/ } \\
\text { female }\end{array}$ & Astrocytoma & Midbrain & Chronic & $\begin{array}{l}\text { Excellent } \\
\text { response }\end{array}$ \\
\hline Lin et al [12] (2010) & $\begin{array}{l}81 \text { years/ } \\
\text { male }^{+}\end{array}$ & B cell lymphoma & Midbrain & Acute & No response \\
\hline Hatano et al [13] (2011) & 70 year/male & B cell lymphoma & $\begin{array}{l}\text { Midbrain, hypothalamus, pineal body, thalamus, } \\
\text { pallidum }\end{array}$ & Acute & $\begin{array}{l}\text { No } \\
\text { administration }\end{array}$ \\
\hline $\begin{array}{l}\text { Wächter et al [14] } \\
\text { (2011) }\end{array}$ & 74 years/male & Astrocytoma & Pons, midbrain, thalamus & Chronic & $\begin{array}{l}\text { Moderate } \\
\text { response }\end{array}$ \\
\hline Present patient & 68 years/male & NKTL & Pons, midbrain, cerebellum cerebral white matter & Acute & No response \\
\hline
\end{tabular}

*Reference number.

${ }^{+}$Complication of obstructive hydrocephalus. 
patient supported the concept that damage of the ponto-mesencephalic tegmentum could contribute to the pathogenesis of PA.

\section{Conclusion}

We highlighted the clinicoradiological course in a patient with transient PA and CNS metastases of NKTL. The neuroradiological changes of the present patient suggest that physicians should pay more attention to fulminant progression of CNS metastases in NKTL patients. MTX pulse therapy dramatically improved the serious neurological deficits and brain lesions in our patient.

\section{Consent}

Informed consent was obtained from the patient and his spouse for publication of this case report and any accompanying images.

\section{Author details}

${ }^{1}$ Department of Hematology and Oncology, Toho University Omori Medical Center, Tokyo, Japan. ${ }^{2}$ Department of Neurology, Toho University Omori Medical Center, Tokyo, Japan. ${ }^{3}$ Division of Pathology, Saiseikai Yokohamashi Tobu Hospital, Yokohama, Japan.

\section{Authors' contributions}

$\mathrm{KI}$ participated in the clinicoradiological studies, its design and drafted the manuscript. SI and KK carried out the clinical evaluation of the patient and wrote the patient case. OK carried out the neuroradiological studies. RS carried out the pathological analysis. Yl participated in coordination and helped to draft the manuscript. All authors read and approved the final manuscript.

\section{Competing interests}

The authors declare that they have no competing interests.

Received: 1 August 2011 Accepted: 2 November 2011

Published: 2 November 2011

\section{References}

1. Barbeau A: Contribution of levodopa therapy to the ncuropharmachology of amnesia. In Parkinson's Disease. Edited by: Siegfried J. Verlag Hans Huber, Bern; 1972:151-159.

2. Imai H, Narabayashi H: Akinesia-concerning 2 cases of pure akinesia. Adv Neurol Sci (Tokyo) 1974, 18:787-794

3. Narabayashi H, Imai H, Yokochi M, Hirayama K, Nakamura R: Cases of pure akinesia without rigidity and tremor and with no effect by L-dopa therapy. In Advances in Parkinsonism. Edited by: Birkmayer W, Hornykiewicz O. Roche, Basel; 1976:335-342.

4. Suzuki T, Yamamoto M, Saitoh M, Aoki A, Imai H, Narabayashi H: A case of intracranial malignant lymphoma with pure akinesia and repeated regression on CT scans. Brain and Nerve 1984, 36:689-696.

5. Pramstaller PP, Salerno A, Bhatia KP, Prugger M, Marsden CD: Primary central nervous system lymphoma presenting with a parkinsonian syndrome of pure akinesia. J Neurol 1999, 246:934-938.

6. Krauss JK, Nobbe F, Wakhloo AK, Mohadjer M, Vach W, Mundinger F: Movement disorders in astrocytomas of the basal ganglia and the thalamus. J Neurol Neurosurg Psychiatry 1992, 55:1162-1167.

7. Krauss JK, Paduch T, Mundinger F, Seeger W: Parkinsonism and rest tremor secondary to supratentorial tumours sparing the basal ganglia. Acta Neurochir (Wien) 1995, 133:22-29.

8. Sánchez-Guerra M, Cerezal L, Leno C, Díez C, Figols J, Berciano J: Primary brain lymphoma presenting as Parkinson's disease. Neuroradiology 2001, 43:36-40.
9. Gherardi R, Roualdes B, Fleury J, Prost C, Poirier J, Degos JD: Parkinsonian syndrome and central nervous system lymphoma involving the substantia nigra. A case report. Acta Neuropathol 1985, 65:338-343.

10. Cicarelli G, Pellecchia MT, Maiuri F, Barone P: Brain stem cystic astrocytoma presenting with "pure" parkinsonism. Mov Disord 1999, 14:364-366,

11. Yoshimura M, Yamamoto T, Iso-o N, Imafuku I, Momose T, Shirouzu I, Kwak S, Kanazawa I: Hemiparkinsonism associated with a mesencephalic tumor. J Neurol Sci 2002, 197:89-92.

12. Lin $C M$, Hong $K$ : Cerebral infratentorial large B-cell lymphoma presenting as Parkinsonism. Tohoku J Exp Med 2010, 220:187-190.

13. Hatano T, Oji Y, Takanashi M, Sasaki M, Ishii H, Hattori N: Case of the Month April/May 2011.[http://www.movementdisorders.org/].

14. Wächter T, Engeholm M, Bisdas S, Schittenhelm J, Gasser T, Krüger R: Slowly progressive Parkinson syndrome due to thalamic butterfly astrocytoma. Neurology 2011, 77:404-405.

15. Freilich RJ, DeAngelis LM: Primary central nervous system lymphoma. Neurol Clin 1995, 13:901-914.

16. Ho FCS, Todd D, Loke SL, Ng RP, Khoo RKK: Clinicopathological features of malignant lymphomas in 294 Hong Kong Chinese patients, retrospective study covering an eight-year period. Int J Cancer 1984, 34:143-148.

17. Liang R, Todd D, Chan TK, Chiu E, Lie A, Kwong YL, Choy D, Ho FC: Treatment outcome and prognostic factors for primary nasal lymphoma. J Clin Oncol 1995, 13:666-670.

18. Yeh KH, Lien HC, Hsu SM, Cheng AL: Quiescent nasal T/NK cell lymphoma manifested as primary central nervous system lymphoma. Am J Hematol 1999, 60:161-163.

19. Fischer L, Koch A, Schlegel U, Koch HC, Wenzel R, Schröder N, Thiel E, Korfel A: Non-enhancing relapse of a primary CNS lymphoma with multiple diffusion-restricted lesions. J Neurooncol 2011, 102:163-166.

20. Barajas RF Jr, Rubenstein JL, Chang JS, Hwang J, Cha S: Diffusion-weighted MR imaging derived apparent diffusion coefficient is predictive of clinical outcome in primary central nervous system lymphoma. AJNR Am J Neuroradiol 2010, 31:60-66.

21. Honma Y, Takahashi H, Takeda S, Ikuta F: An autopsy case of progressive supranuclear palsy showing "pure akinesia without rigidity and tremor and with no effect by L-Dopa therapy". Brain and Nerve 1987, 39:183-187.

22. Williams DR, Holton JL, Strand K, Revesz T, Lees AJ: Pure akinesia with gait freezing: a third clinical phenotype of progressive supranuclear palsy. Mov Disord 2007, 22:2235-2241

23. Facheris MF, Maniak S, Scaravilli F, Schüle B, Klein C, Pramstaller PP: Pure akinesia as initial presentation of PSP: a clinicopathological study. Parkinsonism Relat Disord 2008, 14:517-519.

24. Taniwaki T, Hosokawa S, Goto I, Fujii N, Otsuka M, Kuwabara Y, Ichiya Y, Hasuo K, Kato M: Positron emission tomography (PET) in "pure akinesia". J Neurol Sci 1992, 107:34-39.

25. Park HK, Kim JS, Im KC, Oh SJ, Kim MJ, Lee JH, Chung SJ, Lee MC: Functional brain imaging in pure akinesia with gait freezing: [18F] FDG PET and [18F] FP-CIT PET analyses. Mov Disord 2009, 24:237-245.

26. Lewerenz J, Zurowski B, Jenicke L, Bäumer T, Lees AJ, Münchau A: Lesion of the dorsorostral midbrain sparing the nigrostriatal tract mimics axial rigidity seen in progressive supranuclear palsy. Mov Disord 2005, 20:1071-1075.

\section{Pre-publication history}

The pre-publication history for this paper can be accessed here: http://www.biomedcentral.com/1471-2377/11/137/prepub

doi:10.1186/1471-2377-11-137

Cite this article as: Ishihara et al:: Clinicoradiological changes of brain $\mathrm{NK} / \mathrm{T}$ cell lymphoma manifesting pure akinesia: a case report. BMC Neurology 2011 11:137. 